\title{
Chapter 13 \\ Mean-Field Analysis of Sourlas Codes with Adiabatic Reverse Annealing
}

\author{
Shunta Arai
}

\begin{abstract}
In this chapter, we analyze the typical performance of adiabatic reverse annealing (ARA) for Sourlas codes. Sourlas codes are representative error-correcting codes related to $p$-body spin-glass models and have a first-order phase transition for $p>2$, which degrades the estimation performance. In the ARA formulation, we introduce the initial Hamiltonian which incorporates the prior information of the solution into a vanilla quantum annealing (QA) formulation. The ground state of the initial Hamiltonian represents the initial candidate solution. To avoid the first-order phase transition, we apply ARA to Sourlas codes. We evaluate the typical ARA performance for Sourlas codes using the replica method. We show that ARA can avoid the first-order phase transition if we prepare for the proper initial candidate solution.
\end{abstract}

\subsection{Introduction}

Problems in information processing have been studied analytically from the viewpoint of statistical mechanics [12]. Associative memory, Sourlas codes, code-division multiple-access (CDMA), and image restoration are very popular examples [5, 6, 21, 24]. Many studies have focused on the degradation of the original signal or information due to noise. The noise can be physically regarded as thermal fluctuations. The original information can be estimated from the degraded data by tuning the strength of thermal fluctuations.

In this chapter, we focus mainly on error-correcting codes such as Sourlas codes, which are described by $p$-body spin-glass problems [21]. The main idea of errorcorrecting codes is to add redundancy while sending information to decode the original signal from noisy outputs. In Sourlas codes, the original signal is encoded in the interactions of the spins. To estimate the original signal, we search the ground

\footnotetext{
S. Arai $(\bowtie)$

Graduate School of Information Sciences, Tohoku University, 980-8579 Sendai, Japan

e-mail: shunta.arai.d8@tohoku.ac.jp
}

Sigma-i Co.,Ltd., 108-0075 Minato, Tokyo, Japan

(C) The Author(s) 2022

N. Katoh et al. (eds.), Sublinear Computation Paradigm, https://doi.org/10.1007/978-981-16-4095-7_13 
state of the Hamiltonian or compute the expectation value over the Gibbs-Boltzmann distribution at a finite temperature.

In addition to thermal fluctuations, quantum fluctuations can also be used to infer the original information. Several studies have demonstrated that quantum fluctuations such as the transverse field do not necessarily enhance the performance of decoding for image restoration, Sourlas codes, or CDMA $[2,6,15,16]$. The optimal estimation performance using quantum fluctuations is inferior to that using thermal fluctuations in Bayes-optimal cases. However, in some non-Bayes optimal cases, the estimation performance using finite quantum fluctuations and thermal fluctuations surpasses that using only thermal fluctuations; for example, when the assigned temperature is lower than the true noise scale. This implies the potential of combining quantum and thermal fluctuations for signal recovery problems.

Signal estimation algorithms using quantum fluctuations are related to optimization algorithms using quantum fluctuations, which is known as quantum annealing (QA) [9] or adiabatic quantum computation (AQC) [3]. The QA algorithm is physically implemented in the quantum annealer [7]. The quantum annealer has been tested in numerous applications, including traffic optimization [11] and in vehicles in factories [14].

In a closed system, the QA procedure is as follows. First, we set the initial state as the trivial ground state of the transverse field term. Next, we gradually decrease the strength of the transverse field. Following the Schrodinger equation, the trivial ground state evolves adiabatically into a nontrivial ground state of the target Hamiltonian, which is consistent with a solution of combinatorial optimization problems. The quantum adiabatic theorem indicates that the total computational time for searching the ground state is characterized by the minimum energy gap between the ground state and first excited state [23]. When the target Hamiltonian has a first-order phase transition, the computational time to find the ground state grows exponentially.

Reverse annealing (RA) is a protocol for restarting quantum dynamics from the final state of the standard QA procedure [17]. The RA algorithm can be used to avoid or mitigate the first-order phase transition and is classified into two methods: adiabatic reverse annealing (ARA) [13] and iterated reverse annealing (IRA) [26]. ARA and IRA are distinguished by how the final state is utilized. One implements the final state by introducing the initial Hamiltonian, and the other incorporates it as the initial condition.

In a recent study [2], ARA is applied to CDMA multiuser detection. ARA can avoid or mitigate the first-order phase transition in the CDMA model. In this chapter, we apply ARA for Sourlas codes. Sourlas codes have a first-order phase transition for $p>2$. The existence of the first-order phase transition deteriorates the estimation performance. We evaluate the typical performance of ARA for Sourlas codes using the replica method. We demonstrate that ARA can avoid the first-order phase transition of Sourlas codes if we prepare the proper initial conditions. 


\subsection{Sourlas Codes Using Quantum Fluctuations}

Following a previous study [15], we formulate Sourlas codes using quantum fluctuations. Sourlas codes are set up to send a set of products of $p$ spins $J_{i_{1} \ldots i_{p}}=\xi_{i_{1}} \ldots \xi_{i_{p}}$ through a channel. The symbol $\xi_{i}= \pm 1(i=1 \ldots N)$ represents the original signal, which is independently generated from the uniform distribution $P\left(\xi_{i}\right)=1 / 2$. We consider the Gauss channel as

$$
P\left(J_{i_{1} \ldots i_{p}} \mid\{\xi\}\right)=\left(\frac{N^{p-1}}{J^{2} \pi p !}\right)^{\frac{1}{2}} \exp \left\{-\frac{N^{p-1}}{J^{2} p !}\left(J_{i_{1} \ldots i_{p}}-\frac{J_{0} p ! \xi_{i_{1}} \ldots \xi_{i_{p}}}{N^{p-1}}\right)^{2}\right\}
$$

where $J$ and $J_{0}$ are hyperparameters. The ratio $J_{0} / J$ represents the signal-to-noise ratio. The distribution $P\left(J_{i_{1} \ldots i_{p}} \mid\{\xi\}\right)$ is the conditional probability of the signal $J_{i_{1} \ldots i_{p}}$ for the encoded signal $\xi_{i_{1}} \ldots \xi_{i_{p}}$. We infer the original signal $\{\xi\}$ from the noisy outputs $\left\{J_{i_{1} \ldots i_{p}}\right\}$. Using the Bayes formula, we introduce the posterior probability for the estimated signal $\sigma=\left\{\sigma_{1} \ldots \sigma_{N}\right\} \in\{ \pm 1\}^{N}$ as

$$
P\left(\sigma \mid\left\{J_{i_{1} \ldots i_{p}}\right\}\right)=\frac{P\left(\left\{J_{i_{1} \ldots i_{p}}\right\} \mid \sigma\right) P(\sigma)}{\sum_{\sigma} P\left(\left\{J_{i_{1} \ldots i_{p}}\right\} \mid \sigma\right) P(\sigma)},
$$

where $P\left(\left\{J_{i_{1} \ldots i_{p}}\right\} \mid \sigma\right)$ and $P(\sigma)$ are the likelihood and prior distribution, respectively. The summation of spin variables $\sum_{\sigma}$ is defined for all possible configurations. The likelihood can be expressed as

$$
P\left(\left\{J_{i_{1} \ldots i_{p}}\right\} \mid \boldsymbol{\sigma}\right) \propto \exp \left(\beta \sum_{i_{1}<\cdots<i_{p}} J_{i_{1} \ldots i_{p}} \sigma_{i_{1}} \ldots \sigma_{i_{p}}\right)
$$

where $\beta$ is the inverse temperature and the summation $\sum_{i_{1}<\cdots<i_{p}}$ runs over all possible combinations of $p$ spins out of $N$ spins. According to Eqs. (13.2) and (13.3), the posterior distribution can be written by using the Gibbs-Boltzmann distribution with the classical Hamiltonian $\mathcal{H}(\boldsymbol{\sigma})$, as follows:

$$
\begin{aligned}
P\left(\boldsymbol{\sigma} \mid\left\{J_{i_{1} \ldots i_{p}}\right\}\right) & =\frac{1}{Z} \exp \left\{-\beta\left(\mathcal{H}(\boldsymbol{\sigma})+\mathcal{H}_{\text {init }}(\boldsymbol{\sigma})\right)\right\} \\
Z & =\sum_{\sigma} \exp \left\{-\beta\left(\mathcal{H}(\boldsymbol{\sigma})+\mathcal{H}_{\text {init }}(\boldsymbol{\sigma})\right)\right\} \\
\mathcal{H}(\boldsymbol{\sigma}) & =-\sum_{i_{1}<\cdots<i_{p}} J_{i_{1} \ldots i_{p}} \sigma_{i_{1}} \ldots \sigma_{i_{p}}
\end{aligned}
$$

where $Z$ is the partition function and $\mathcal{H}_{\text {init }}(\boldsymbol{\sigma})$ is the initial Hamiltonian, which represents the prior information of the estimated signal. We generally assume that the prior of the estimated signal follows a uniform distribution $P(\sigma)=1 / 2^{N}$. 
To decode the original signal, one decoding strategy is the maximum a posteriori (MAP) estimation, which corresponds to searching the ground state of the classical Hamiltonian of Sourlas codes in the limit of zero temperature. Another is the marginal posterior mode (MPM) estimation, which corresponds to finding the expectation value over the posterior distribution at a finite temperature. In the limit of zero temperature, the MPM estimation is consistent with the MAP estimation. In this chapter, we mainly consider the MPM estimation. The estimation performance can be evaluated by the overlap between the original and estimated signal as

$$
\mathcal{M}(\beta)=\operatorname{Tr}_{\xi} \prod_{i_{1}<\ldots<i_{p}} \int d J_{i_{1} \ldots i_{p}} P(\xi) P\left(\left\{J_{i_{1} \ldots i_{p}}\right\} \mid \xi\right) \xi_{i} \operatorname{sgn}\left\langle\sigma_{i}\right\rangle
$$

where $\langle\cdot\rangle$ is the expectation over the posterior distribution $P\left(\sigma \mid\left\{J_{i_{1} \ldots i_{p}}\right\}\right)$. This quantity is expected to exhibit a "self-averaging" property in the thermodynamics limit $N \rightarrow \infty$. This means that the observables, such as the overlap for a quenched realization of the data $\left\{J_{i_{1} \ldots i_{p}}\right\}$, and $\boldsymbol{\xi}$, are equivalent to the expectation itself over the data distribution $P(\xi) P\left(\left\{J_{i_{1} \ldots i_{p}}\right\} \mid \xi\right)$. In this case, the overlap can be expressed as $\lim _{N \rightarrow \infty} M=\left[\xi_{i} \operatorname{sgn}\left\langle\sigma_{i}\right\rangle\right]$, where the bracket [.] indicates the expectation over the data distribution.

Quantum fluctuations can be utilized to decode the original information. The Hamiltonian of Sourlas code using quantum fluctuations is expressed as follows:

$$
\begin{aligned}
\hat{\mathcal{H}} & =s \hat{\mathcal{H}}_{0}+(1-s) \hat{\mathcal{H}}_{\mathrm{TF}}, \\
\hat{\mathcal{H}}_{0} & =-\sum_{i_{1}<\cdots<i_{p}} J_{i_{1} \ldots i_{p}} \hat{\sigma}_{i_{1}}^{z} \ldots \hat{\sigma}_{i_{p}}^{z}, \\
\hat{\mathcal{H}}_{\mathrm{TF}} & =-\sum_{i=1}^{N} \hat{\sigma}_{i}^{x},
\end{aligned}
$$

where $\hat{\sigma}_{i}^{z}$ and $\hat{\sigma}_{i}^{x}$ are the $z$ and $x$ components of the Pauli matrix at site $i$. We parameterize the Hamiltonian by the annealing parameter $s$ for the ARA formulation. Note that $\hat{\mathcal{H}}_{0}$ and $\hat{\mathcal{H}}_{\mathrm{TF}}$ consist of the $z$ and $x$ components of the Pauli matrices, respectively. As in the classical case, we can consider the MPM estimation using quantum fluctuations. The performance of the MPM estimation using quantum fluctuations can be evaluated by the overlap as follows:

$$
\begin{aligned}
M(\beta, s) & =\operatorname{Tr}_{\{\xi\}} \int \prod_{i_{1}<\cdots<i_{p}} d J_{i_{1} \ldots i_{p}} P\left(\left\{J_{i_{1} \ldots i_{p}}\right\} \mid\{\xi\}\right) P(\{\xi\}) \xi_{i} \operatorname{sgn}\left\langle\hat{\sigma}_{i}^{z}\right\rangle_{\mathrm{TF}} \\
& \equiv\left[\xi_{i} \operatorname{sgn}\left(\left\langle\hat{\sigma}_{i}^{z}\right\rangle_{\mathrm{TF}}\right)\right]
\end{aligned}
$$

where $\langle(\cdot)\rangle_{\mathrm{TF}} \equiv \operatorname{Tr}((\cdot) \hat{\rho})$ denotes the expectation over the density matrix $\hat{\rho} \equiv$ $e^{-\beta \hat{\mathcal{H}}} / \operatorname{Tr} e^{-\beta \hat{\mathcal{H}}}$. 


\subsection{Replica Analysis for Adiabatic Reverse Annealing}

Following Ref. [13], we formulate Sourlas codes using quantum fluctuations in ARA as follows:

$$
\begin{aligned}
\hat{\mathcal{H}} & =s \hat{\mathcal{H}}_{0}+(1-s)(1-\lambda) \hat{\mathcal{H}}_{\text {init }}+(1-s) \lambda \hat{\mathcal{H}}_{\mathrm{TF}}, \\
\hat{\mathcal{H}}_{\text {init }} & =-\sum_{i=1}^{N} \tau_{i} \hat{\sigma}_{i}^{z},
\end{aligned}
$$

where $\lambda(0 \leq \lambda \leq 1)$ is the RA parameter. We now introduce the initial candidate solution $\tau_{i}= \pm 1$ that is expected to be close to the correct ground state $\xi_{i}$. We define the probability distribution of the initial candidate solutions as follows:

$$
P(\boldsymbol{\tau})=\prod_{i=1}^{N} P\left(\tau_{i}\right)=\prod_{i=1}^{N}\left(c_{1} \delta\left(\tau_{i}-\xi_{i}\right)+c_{-1} \delta\left(\tau_{i}+\xi_{i}\right)\right),
$$

where we utilize the symbol $c_{1}=c$ and $c_{-1}=1-c$. The number $c(0 \leq c \leq 1)$ denotes the fraction of the original signal $\tau_{i}=\xi_{i}$ in the initial candidate solution as

$$
c=\frac{1}{N} \sum_{i=1}^{N} \delta_{\tau_{i} \xi_{i}}
$$

We consider that the ARA formulation is the case when we adopt $P\left(\sigma^{z} \mid \tau\right) \propto$ $\exp \left(-\beta \hat{H}_{\text {init }}\right)$ as the prior distribution.

The typical behaviors of the order parameters, such as the overlap, can be obtained via the free energy. The free energy density $f$ can be evaluated as $-\beta f=\lim _{N \rightarrow \infty}(1 / N)[\ln Z]$ in the limit of $N \rightarrow \infty$ where $Z=\operatorname{Tr} \exp (-\beta \hat{\mathcal{H}})$ is the partition function of Eq. (13.12). In general, the direct computation of the free energy density is hard due to the configuration average of $\ln Z$ and the offdiagonal elements in Eq. (13.12). The configuration average can be found using the replica trick [20]. Even though we can avoid the direct computation of [ $\ln Z]$, we cannot apply the standard techniques to evaluate the free energy density due to the non-commutativity of the Hamiltonian.

First, to eliminate the non-commutativity of the Hamiltonian, we apply the Suzuki-Trotter decomposition [22] to the partition function:

$$
\begin{aligned}
Z & =\lim _{M \rightarrow \infty} \operatorname{Tr}\left\{\exp \left(-\frac{\beta}{M}\left(s \hat{\mathcal{H}}_{0}+(1-s)(1-\lambda) \hat{\mathcal{H}}_{\text {init }}\right)\right) \exp \left(-\frac{\beta(1-s) \lambda}{M} \hat{\mathcal{H}}_{\mathrm{TF}}\right)\right\}^{M} \\
& =\lim _{M \rightarrow \infty} Z_{M}
\end{aligned}
$$

where 


$$
\begin{aligned}
Z_{M}= & \operatorname{Tr} \exp \left(\frac{\beta s}{M} \sum_{t=1}^{M} \sum_{i_{1}<\cdots<i_{p}} J_{i_{1} \cdots p} \sigma_{i_{1}}^{z}(t) \ldots \sigma_{i_{p}}^{z}(t)+\frac{\beta(1-s)(1-\lambda)}{M} \sum_{i=1}^{N} \tau_{i} \sigma_{i}^{z}(t)\right. \\
& \left.+\frac{\beta(1-s) \lambda}{M} \sum_{i=1}^{N} \sigma_{i}^{x}(t)\right) \times \prod_{i=1}^{N} \prod_{t=1}^{M}\left\langle\sigma_{i}^{z}(t) \mid \sigma_{i}^{x}(t)\right\rangle\left\langle\sigma_{i}^{x}(t) \mid \sigma_{i}^{z}(t+1)\right\rangle,
\end{aligned}
$$

where the symbol $t$ is the index of the Trotter slice, $M$ is the Trotter number, and $\operatorname{Tr}$ denotes the trace in the $z$ and $x$ basis. We impose the periodic boundary conditions $\sigma_{i}^{z}(1)=\sigma_{i}^{z}(M+1)$ for all $i$ and introduce the identity operator $\hat{1}=\sum_{\left\{\sigma^{z}(t)\right\}}\left|\left\{\sigma^{z}(t)\right\}\right\rangle\left\langle\left\{\sigma^{z}(t)\right\}\right|$ and $\hat{1}=\sum_{\left\{\sigma^{x}(t)\right\}}\left|\left\{\sigma^{x}(t)\right\}\right\rangle\left\langle\left\{\sigma^{x}(t)\right\}\right|$. The detailed calculation is given in Appendix 13.5.

To evaluate $[\ln Z$ ], we utilize the replica trick [20]:

$$
[\log Z]=\lim _{n \rightarrow 0} \frac{\left[Z^{n}\right]-1}{n}
$$

where $n$ is the replica number. The replicated partition function can be written as

$$
\begin{aligned}
& {\left[Z^{n}\right]=\lim _{M \rightarrow \infty} \sum_{\left\{\xi_{i}= \pm 1\right\}} \sum_{\left\{\tau_{i}= \pm \xi_{i}\right\}} P(\xi) P(\boldsymbol{\tau}) \prod_{i_{1}<\cdots<i_{p}} \int d J_{i_{1}, \ldots, i_{p}} P\left(\left\{J_{i_{1}, \ldots, i_{p}}\right\} \mid \xi_{i_{1}} \ldots \xi_{i_{p}}\right)} \\
& \times \operatorname{Tr} \exp \left\{\frac{\beta s}{M} \sum_{t, a} \sum_{i_{1}<\cdots<i_{p}} J_{i_{1} \cdots p_{p}} \sigma_{i_{1} a}^{z}(t) \ldots \sigma_{i_{p} a}^{z}(t)+\frac{\beta(1-s)(1-\lambda)}{M} \sum_{i, t, a} \tau_{i} \sigma_{i a}^{z}(t)\right. \\
& \left.+\frac{\beta(1-s) \lambda}{M} \sum_{i, t, a} \sigma_{i a}^{x}(t)\right\} \prod_{i, t, a}\left\langle\sigma_{i a}^{z}(t) \mid \sigma_{i a}^{x}(t)\right\rangle\left\langle\sigma_{i a}^{x}(t) \mid \sigma_{i a}^{z}(t+1)\right\rangle,
\end{aligned}
$$

in which $a$ denotes the replica index.

To remove the dependency of the original signal $\{\xi\}$, we apply the gauge transformation $J_{i_{1} \ldots i_{p}} \rightarrow J_{i_{1} \ldots i_{p}} \xi_{i_{1}} \ldots \xi_{i_{p}}$ and $\sigma_{i a}^{z}(t) \rightarrow \sigma_{i a}^{z}(t) \xi_{i}$ to the partition function $\left[Z_{M}^{n}\right]$. Performing the Gaussian integration over the distribution in Eq. (13.1), we introduce the following order parameters as

$$
\begin{aligned}
m_{a}(t) & =\frac{1}{N} \sum_{i=1}^{N} \sigma_{i a}^{z}(t), \\
q_{a b}\left(t, t^{\prime}\right) & =\frac{1}{N} \sum_{i=1}^{N} \sigma_{i a}^{z}(t) \sigma_{i b}^{z}\left(t^{\prime}\right), \\
R_{a}\left(t, t^{\prime}\right) & =\frac{1}{N} \sum_{i=1}^{N} \sigma_{i a}^{z}(t) \sigma_{i a}^{z}\left(t^{\prime}\right),
\end{aligned}
$$




$$
m_{a}^{x}(t)=\frac{1}{N} \sum_{i=1}^{N} \sigma_{i a}^{x}(t) .
$$

The physical meanings of the order parameters are as follows: $m_{a}(t)$ is the magnetization, $q_{a b}\left(t, t^{\prime}\right)$ is the spin-glass order parameter, $R_{a}\left(t, t^{\prime}\right)$ is the correlation between each Trotter slice, and $m_{a}^{x}(t)$ is the transverse magnetization. Moreover, we introduce the auxiliary parameters $\tilde{m}_{a}(t), \tilde{q}_{a b}\left(t, t^{\prime}\right), \tilde{R}_{a}\left(t, t^{\prime}\right), \tilde{m}_{a}^{x}(t)$ of the order parameters with the delta function and its Fourier integral representation. Under the replica symmetry (RS) ansatz and static approximation, $m_{a}(t)=m, q_{a b}\left(t, t^{\prime}\right)=$ $q, R_{a}\left(t, t^{\prime}\right)=R, m_{a}^{x}(t)=m^{x}, \tilde{m}_{a}(t)=\tilde{m}, \tilde{q}_{a b}\left(t, t^{\prime}\right)=\tilde{q}, \tilde{R}_{a}\left(t, t^{\prime}\right)=\tilde{R}, \tilde{m}_{a}^{x}(t)=$ $\tilde{m}^{x}$, we can attain the RS free energy density:

$$
\begin{aligned}
-\beta f_{\mathrm{RS}} & =\beta s J_{0} m^{p}+\frac{\beta^{2} s^{2} J^{2}}{4}\left(R^{p}-q^{p}\right)+\beta(1-s) \lambda m^{x}-\beta m \tilde{m}-\beta m^{x} \tilde{m}^{x} \\
& -\frac{\beta^{2}}{2}(R \tilde{R}-q \tilde{q})+\sum_{a= \pm 1} c_{a} \int D z \ln 2 Y_{a} \\
Y_{a} & \equiv \int D y \cosh \beta u_{a} \\
u_{a} & \equiv \sqrt{g_{a}^{2}+\left(\tilde{m}^{x}\right)^{2}} \\
g_{a} & \equiv \tilde{m}+a(1-s)(1-\lambda)+\sqrt{\tilde{q}} z+\sqrt{\tilde{R}-\tilde{q}} y
\end{aligned}
$$

where $D z$ means that the Gaussian measure $D z:=1 / \sqrt{2 \pi} d z e^{-z^{2} / 2}$, and $D y$ is the same as $D z$. Detailed calculations for deriving the free energy density in Eq. (13.24) are provided in Appendix 13.5. The order parameters and their auxiliary parameters are determined by the saddle-point conditions in the free energy density. The extremization of Eq. (13.24) yields the following saddle-point equations:

$$
\begin{gathered}
m=\sum_{a= \pm 1} c_{a} \int D z Y_{a}^{-1} \int D y\left(\frac{g_{a}}{u_{a}}\right) \sinh \beta u_{a} \\
q=\sum_{a= \pm 1} c_{a} \int D z\left\{Y_{a}^{-1} \int D y\left(\frac{g_{a}}{u_{a}}\right) \sinh \beta u_{a}\right\}^{2} \\
R=\sum_{a= \pm 1} c_{a} \int D z Y_{a}^{-1} \int D y\left\{\left(\frac{\left(\tilde{m}^{x}\right)^{2}}{\beta u_{a}^{3}}\right) \sinh \beta u_{a}+\left(\frac{g_{a}}{u_{a}}\right)^{2} \cosh \beta u_{a}\right\} \\
m^{x}=\sum_{a= \pm 1} c_{a} \int D z Y_{a}^{-1} \int D y\left(\frac{\tilde{m}^{x}}{u_{a}}\right) \sinh \beta u_{a}
\end{gathered}
$$




$$
\begin{aligned}
\tilde{m} & =s J_{0} p m^{p-1}, \\
\tilde{q} & =\frac{s^{2} J^{2}}{2} p q^{p-1}, \\
\tilde{R} & =\frac{s^{2} J^{2}}{2} p R^{p-1}, \\
\tilde{m}^{x} & =(1-s) \lambda .
\end{aligned}
$$

From Eq. (13.11), the overlap function is easily expressed as

$$
M(\beta, s, \lambda)=\sum_{a= \pm 1} c_{a} \int D z \operatorname{sgn}\left\{Y_{a}^{-1} \int D y\left(\frac{g_{a}}{u_{a}}\right) \sinh \beta u_{a}\right\} .
$$

In the low-temperature region, the $p$-body spin-glass model is known to exhibit replica symmetry breaking (RSB) [4]. The stability condition of RS solutions under the static approximation is expressed as

$$
\begin{aligned}
& \frac{\beta^{2} s^{2} J^{2} p(p-1)}{2} q^{p-2}\left(\sum_{a= \pm 1} c_{a} A_{a}\right)<1 \\
A_{a} & \equiv \int D z\left\{\left(Y_{a}^{-1} \int D y\left(\frac{g_{a}}{u_{a}}\right) \sinh \beta u_{a}\right)^{2}\right. \\
& \left.-Y_{a}^{-1}\left(\int D y\left(\frac{\left(\tilde{m}^{x}\right)^{2}}{\beta u_{a}^{3}}\right) \sinh \beta u_{a}+\int D y\left(\frac{g_{a}}{u_{a}}\right)^{2} \cosh \beta u_{a}\right)\right\}^{2} .
\end{aligned}
$$

This condition, called the Almeida-Thouless (AT) condition [1], can be attained by considering perturbations to the RS solutions. This result is consistent with the previous result in Ref. [25] for $p=2, J_{0}=0$, and $\lambda=1$.

\subsection{Numerical Experiments}

We numerically solve the saddle-point equations in Eqs. (13.28)-(13.35) with $p=5$, temperature $T=0.05$, and signal-to-noise ratio $J_{0} / J=1.5$. To evaluate the typical MPM estimation performance, we often utilize the overlap $M(\beta, s, \lambda)$. In this chapter, we focus mainly on the possibility of avoiding the first-order phase transition by ARA. For the sake of simplicity and computational cost, we adopt the magnetization as a measure of the average MPM estimation performance using ARA. Figure 13.1a shows the phase diagram of the Sourlas codes using quantum fluctuations in ARA. We consider three initial conditions: $c=0.7,0.8$, and 0.95. Each line represents a point of the first-order phase transition. We call these lines "critical" lines. We can avoid a first-order phase transition by preparing for proper initial conditions. When 


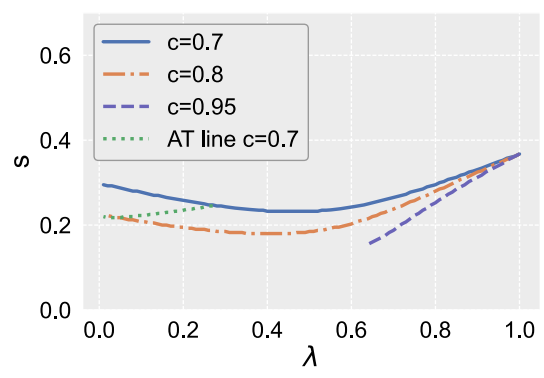

(a)

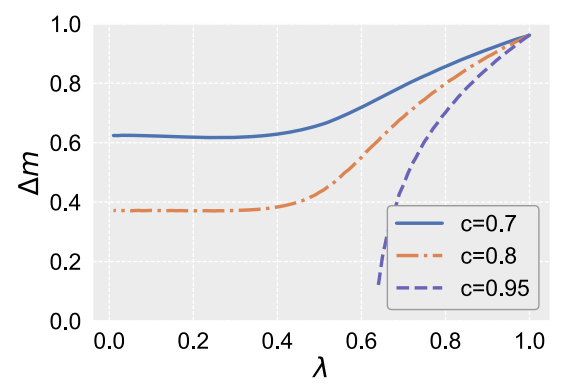

(b)

Fig. 13.1 a Phase diagram of Sourlas codes in ARA for $c=0.7,0.8$, and 0.95. The vertical and horizontal axes represent the annealing parameter and the RA parameter, respectively. Each line represents the point where the first-order phase transition occurs. The AT line indicates where the AT condition is broken above the line. b Differences in magnetization between two local minima at the first-order phase transition in Fig. 13.1 (a). The vertical axis denotes the differences in the magnetization between two local minima at the first-order phase transition while the horizontal axis represents the RA parameter

we increase the ratio of the ground state in the initial Hamiltonian, the region where we can avoid the first-order phase transition becomes wider.

We also compute the AT condition Eq. (13.37). As shown in Fig. 13.1a, the AT condition is broken between the AT line and the "critical" line for $c=0.7$. If the fraction of the ground state in the initial candidate solution is not enough, the spinglass phase emerges and RSB occurs. The emergence of RSB implies the existence of a metastable state. Figure 13.1a shows that we can avoid RSB if we tune the RA parameter $\lambda$. For $c=0.8$, the AT condition is broken in the low $\lambda$ region. The region where the AT condition is broken is smaller than that for $c=0.7$. Since we cannot distinguish the AT line from the "critical" line at this scale, we omit the AT line from Fig. 13.1a. For $c=0.95$, the AT condition holds. Therefore, the local stability of the $\mathrm{RS}$ solution is recovered if we can prepare for the proper initial conditions.

To evaluate the extent to which ARA mitigates the difficulty of estimating the original signal, we plot the differences in the magnetization $\Delta m$ between the two local minima at the first-order phase transition for $c=0.7,0.8$, and 0.95. Significant differences in the magnetization result in the separation of the two local minima of the free energy. Figure $13.1 \mathrm{~b}$ shows that $\Delta m$ decreases as $c$ increases. The two local minima of free energy are brought closer by ARA. As discussed in Ref. [13], the quantum tunneling rate between two local minima in the free-energy landscape increases if the distance between the two local minima is smaller. Our results demonstrate that ARA for Sourlas codes enhances the quantum tunneling effects if we prepare for an appropriate initial condition. This result is consistent with the CDMA model [2]. 


\subsection{Summary}

In this chapter, we explained a mean field analysis of ARA for Sourlas codes. Sourlas codes have a first-order phase transition with $p>2$, which deteriorates their estimation performance. To avoid the first-order phase transition, we applied ARA to Sourlas codes. The first-order phase transition can be avoided by preparing for the proper initial conditions. The region where the first-order phase transition can be avoided becomes larger as $c$ increases. We investigated the differences in magnetization between the two local minima at the first-order phase transition. When ARA was applied, the two local minima of the free energy came closer if we prepared for the proper initial conditions. ARA improved the probability of escaping the local minimum by quantum tunneling. This study shows that ARA can be useful for error correcting codes.

In the practical case, we need to prepare for the initial candidate solution by using some algorithms. In the previous study [2] for CDMA multiuser detection, we utilized the approximate message passing algorithm [8] to prepare for the initial candidate solution. The performance of ARA in practical case was different from the oracle cases where the initial candidate solution was generated from the original signal. Evaluation of the performance of ARA in the practical case for Sourlas codes is an interesting future direction.

Acknowledgements We are grateful for valuable comments from Kazuyuki Tanaka, Masayuki Ohzeki, Manaka Okuyama, and ACC Coolen. This work was partly supported by JST-CREST (No. JPMJCR1402).

\section{Appendix 1: Derivation of Eq. (13.17)}

In this appendix, we derive Eq. (13.15) in detail. We mainly follow the references [10, $18,19]$. We consider the $z$ basis as the computational basis. In this case, Tr is replaced by $\sum_{\left\{\sigma^{z}\right\}}\left\langle\left\{\sigma^{z}\right\}|(\cdot)|\left\{\sigma^{z}\right\}\right\rangle$ and $\left|\left\{\sigma^{z}\right\}\right\rangle \equiv \otimes_{i=1}^{N}\left|\sigma_{i}^{z}\right\rangle$. For the $z$ basis, we introduce $M$ copies of the identity operator $\hat{1}=\sum_{\left\{\sigma^{z}(t)\right\}}\left|\left\{\sigma^{z}(t)\right\}\right\rangle\left\langle\left\{\sigma^{z}(t)\right\}\right|$ into Eq. (13.16),

$$
\begin{aligned}
Z_{M} & =\lim _{M \rightarrow \infty} \prod_{t=1}^{M} \sum_{\left\{\sigma^{z}(t)\right\}} \exp \left(-\frac{\beta}{M} \sum_{t=1}^{M}\left(s \mathcal{H}_{0}+(1-s)(1-\lambda) \mathcal{H}_{\text {init }}\right)\right) \\
& \times \prod_{t=1}^{M}\left\langle\left\{\sigma^{z}(t)\right\}\left|\exp \left(-\frac{\beta(1-s) \lambda}{M} \hat{\mathcal{H}}_{\mathrm{TF}}\right)\right|\left\{\sigma^{z}(t+1)\right\}\right\rangle
\end{aligned}
$$

where we introduce the periodic boundary condition $\left|\left\{\sigma^{z}(1)\right\}\right\rangle=\left|\left\{\sigma^{z}(M+1)\right\}\right\rangle$. To show the dependence of the spin operator on the Trotter index, arguments are added to each Hamiltonian in Eq. (13.39). For $x$ basis, we similarly introduce the $M$ copies of the identity operator $\hat{1}=\sum_{\left\{\sigma^{x}(t)\right\}}\left|\left\{\sigma^{x}(t)\right\}\right\rangle\left\langle\left\{\sigma^{x}(t)\right\}\right|$ into Eq. (13.39). The last 
term in Eq. (13.39) can be written as

$$
\prod_{t=1}^{M} \sum_{\left\{\sigma^{x}(t)\right\}} \exp \left(-\frac{\beta(1-s) \lambda}{M} \mathcal{H}_{\mathrm{TF}}\right) \prod_{t=1}^{M}\left\langle\left\{\sigma^{z}(t)\right\} \mid\left\{\sigma^{x}(t)\right\}\right\rangle\left\langle\left\{\sigma^{x}(t)\right\} \mid\left\{\sigma^{z}(t+1)\right\}\right\rangle
$$

Finally, we can obtain Eq. (13.17) in the main text as

$$
\begin{aligned}
Z_{M} & =\prod_{t=1}^{M} \operatorname{Tr} \exp \left(-\frac{\beta}{M} \sum_{t=1}^{M}\left(s \mathcal{H}_{0}+(1-s)(1-\lambda) \mathcal{H}_{\text {init }}\right)-\frac{\beta(1-s) \lambda}{M} \sum_{t=1}^{M} \mathcal{H}_{\mathrm{TF}}\right) \\
& \times \prod_{i=1}^{N} \prod_{t=1}^{M}\left\langle\sigma_{i}^{z}(t) \mid \sigma_{i}^{x}(t)\right\rangle\left\langle\sigma_{i}^{x}(t) \mid \sigma_{i}^{z}(t+1)\right\rangle,
\end{aligned}
$$

where $\operatorname{Tr}$ denotes the summation over all the possible spin configurations $\left\{\sigma_{i}^{z}\right\}$ and $\left\{\sigma_{i}^{x}\right\}$. Since the first term in Eq. (13.41) consists of the commutable numbers, we can take the configuration average over the data distribution.

\section{Appendix 2: Derivation of the RS Free Energy}

We derive the free energy density under the RS ansatz and the static approximation. After the gauge transformation $J_{i_{1} \ldots i_{p}} \rightarrow J_{i_{1} \ldots i_{p}} \xi_{i_{1}} \ldots \xi_{i_{p}}$ and $\sigma_{i a}^{z}(t) \rightarrow \sigma_{i a}^{z}(t) \xi_{i}$, we integrate over $J_{i_{1}, \ldots, i_{p}}$ as

$$
\begin{aligned}
& \prod_{i_{1}<\cdots<i_{p}}\left[\int d J_{i_{1}, \ldots, i_{p}} P\left(\left\{J_{i_{1}, \ldots, i_{p}}\right\} \mid\{\xi\}\right) \exp \left\{\frac{\beta s}{M} \sum_{a, t} J_{i_{1} \ldots p_{p}} \sigma_{i_{1} a}^{z}(t) \ldots \sigma_{i_{p} a}^{z}(t)\right\}\right] \\
= & \prod_{i_{1}<\cdots<i_{p}} \exp \left\{\frac{N^{p-1}}{J^{2} p !}\left(\frac{J_{0} p !}{N^{p-1}}+\frac{\beta s J^{2} p !}{2 M N^{p-1}} \sum_{a, t} \sigma_{i_{1} a}^{z}(t) \ldots \sigma_{i_{p} a}^{z}(t)\right)^{2}-\frac{J_{0}^{2} p !}{J^{2} N^{p-1}}\right\} \\
\simeq & \exp \left\{\frac{\beta s J_{0} N}{M} \sum_{a, t}\left(\frac{1}{N} \sum_{i=1}^{N} \sigma_{i a}^{z}(t)\right)^{p}+\frac{\beta^{2} s^{2} J^{2} N}{4 M^{2}} \sum_{a, b, t, t^{\prime}}\left(\frac{1}{N} \sum_{i=1}^{N} \sigma_{i a}^{z}(t) \sigma_{i b}^{z}\left(t^{\prime}\right)\right)^{p}\right\},
\end{aligned}
$$

where we use the expression $\sum_{i_{1}<\cdots<i_{p}} \sigma_{i_{1}}^{z} \ldots \sigma_{i_{p}}^{z}=\left(N^{p} / p !\right)\left(\sum_{i=1}^{N} \sigma_{i}^{z} / N\right)^{p}+$ $O\left(N^{p-1}\right)$. We introduce the delta function and its Fourier integral representation for Eqs. (13.20)-(13.23) as follows:

$$
\prod_{a, t} \int d m_{a}(t) \delta\left(m_{a}(t)-\frac{1}{N} \sum_{i=1}^{N} \xi_{i} \sigma_{i a}^{z}(t)\right)
$$




$$
\begin{aligned}
& =\prod_{a, t} \int \frac{\beta i N d m_{a}(t) d \tilde{m}_{a}(t)}{2 \pi M} e^{-\frac{\beta \tilde{m}_{a}(t)}{M}\left(N m_{a}(t)-\sum_{i=1}^{N} \xi_{i} \sigma_{i a}^{z}(t)\right)} \\
& \prod_{a, t, t^{\prime}} \int d R_{a}\left(t, t^{\prime}\right) \delta\left(R_{a}\left(t, t^{\prime}\right)-\frac{1}{N} \sum_{i=1}^{N} \sigma_{i a}^{z}(t) \sigma_{i a}^{z}\left(t^{\prime}\right)\right) \\
& =\prod_{a, t, t^{\prime}} \int \frac{\beta^{2} i N d R_{a}\left(t, t^{\prime}\right) d \tilde{R}_{a}\left(t, t^{\prime}\right)}{4 \pi M^{2}} e^{-\frac{\beta^{2} \tilde{R}_{a}\left(t, t^{\prime}\right)}{2 M^{2}}\left(N R_{a}\left(t, t^{\prime}\right)-\sum_{i=1}^{N} \sigma_{i a}^{z}(t) \sigma_{i a}^{z}\left(t^{\prime}\right)\right)} \\
& \prod_{a \neq b, t, t^{\prime}} \int d q_{a b}\left(t, t^{\prime}\right) \delta\left(q_{a b}\left(t, t^{\prime}\right)-\frac{1}{N} \sum_{i=1}^{N} \sigma_{i a}^{z}(t) \sigma_{i b}^{z}\left(t^{\prime}\right)\right) \\
& =\prod_{a \neq b, t, t^{\prime}} \int \frac{\beta^{2} i N d q_{a b}\left(t, t^{\prime}\right) d \tilde{q}_{a b}\left(t, t^{\prime}\right)}{4 \pi M^{2}} e^{-\frac{\beta^{2} \tilde{q}_{a b}\left(t, t^{\prime}\right)}{2 M^{2}}\left(N q_{a b}\left(t, t^{\prime}\right)-\sum_{i=1}^{N} \sigma_{i a}^{z}(t) \sigma_{i b}^{z}\left(t^{\prime}\right)\right)} \\
& \prod_{a, t} \int d m_{a}^{x}(t) \delta\left(m_{a}^{x}(t)-\frac{1}{N} \sum_{i=1}^{N} \sigma_{i a}^{x}(t)\right) \\
& =\prod_{a, t} \int \frac{\beta i N d m_{a}^{x}(t) d \tilde{m}_{a}^{x}(t)}{2 \pi M} e^{-\frac{\beta \tilde{m}_{a}^{x}(t)}{M}\left(N m_{a}^{x}(t)-\sum_{i=1}^{N} \sigma_{i a}^{x}(t)\right)} .
\end{aligned}
$$

The partition function can be written as

$$
\begin{aligned}
{\left[Z^{n}\right] \simeq \lim _{M \rightarrow \infty} \prod_{a, t} \int \frac{\beta i N d m_{a}(t) d \tilde{m}_{a}(t)}{2 \pi M} \prod_{a, t \neq t^{\prime}} \int \frac{\beta^{2} i N d R_{a}\left(t, t^{\prime}\right) d \tilde{R}_{a}\left(t, t^{\prime}\right)}{4 \pi M^{2}} } \\
\times \prod_{a \neq b, t, t^{\prime}} \int \frac{\beta^{2} i N d q_{a b}\left(t, t^{\prime}\right) d \tilde{q}_{a b}\left(t, t^{\prime}\right)}{4 \pi M^{2}} \prod_{a, t} \int \frac{\beta N d m_{a}^{x}(t) d \tilde{m}_{a}^{x}(t)}{2 \pi i M} e^{G 1+G 2+G 3}, \\
e^{G 1} \equiv \exp \left\{\frac{\beta s J_{0} N}{M} \sum_{a, t}\left(m_{a}(t)\right)^{p}+\frac{\beta^{2} s^{2} J^{2} N}{4 M^{2}}\left(\sum_{a \neq b, t, t^{\prime}} q_{a b}^{p}\left(t, t^{\prime}\right)+\sum_{a, t \neq t^{\prime}} R_{a}^{p}\left(t, t^{\prime}\right)+n M\right)\right\} \\
e^{G_{2}} \equiv \sum_{\left\{\xi_{i}= \pm 1\right\}\left\{\tau_{i}= \pm \xi_{i}\right\}} P(\xi) P(\tau) \operatorname{Tr} \exp \left\{\frac{\beta}{M} \sum_{a, t} \tilde{m}_{a}(t) \sum_{i=1}^{N} \sigma_{i a}^{z}(t)\right. \\
+\frac{\beta(1-s)(1-\lambda)}{M} \sum_{a, t, i} \tau_{i} \xi_{i} \sigma_{i a}^{z}(t)+\frac{\beta^{2}}{2 M^{2}} \sum_{a, t \neq t^{\prime}} \tilde{R}_{a}\left(t, t^{\prime}\right) \sum_{i=1}^{N} \sigma_{i a}^{z}(t) \sigma_{i a}^{z}\left(t^{\prime}\right) \\
\left.+\frac{\beta^{2}}{2 M^{2}} \sum_{a \neq b} \sum_{t, t^{\prime}} \tilde{q}_{a b}\left(t, t^{\prime}\right) \sum_{i=1}^{N} \sigma_{i a}^{z}(t) \sigma_{i b}^{z}\left(t^{\prime}\right)+\frac{\beta}{M} \sum_{a, t} \tilde{m}_{a}^{x}(t) \sum_{i=1}^{N} \sigma_{i a}^{x}(t)\right\} \\
\quad \times \prod_{a, t, i}\left\langle\sigma_{i a}^{z}(t) \mid \sigma_{i a}^{x}(t)\right\rangle\left\langle\sigma_{i a}^{x}(t) \mid \sigma_{i a}^{z}(t+1)\right\rangle,
\end{aligned}
$$




$$
\begin{aligned}
e^{G_{3}} & \equiv \exp \left\{-\frac{\beta N}{M} \sum_{a, t} \tilde{m}_{a}(t) m_{a}(t)-\frac{\beta^{2} N}{2 M^{2}} \sum_{a, t \neq t^{\prime}} \tilde{R}_{a}\left(t, t^{\prime}\right) R_{a}\left(t, t^{\prime}\right)\right. \\
& -\frac{\beta^{2} N}{2 M^{2}} \sum_{a<b} \sum_{t, t^{\prime}} \tilde{q}_{a b}\left(t, t^{\prime}\right) q_{a b}\left(t, t^{\prime}\right)-\frac{\beta N}{M} \sum_{a, t} \tilde{m}_{a}^{x}(t) m_{a}^{x}(t) \\
& \left.+\frac{\beta(1-s) \lambda N}{M} \sum_{a, t} m_{a}^{x}(t)\right\} .
\end{aligned}
$$

We assume the RS ansatz and the static approximation as

$$
\begin{aligned}
& m_{a}(t)=m, q_{a b}\left(t, t^{\prime}\right)=q(a \neq b), R_{a}\left(t, t^{\prime}\right)=R\left(t \neq t^{\prime}\right), m_{a}^{x}(t)=m^{x}, \\
& \tilde{m}_{a}(t)=\tilde{m}, \tilde{q}_{a b}\left(t, t^{\prime}\right)=\tilde{q}(a \neq b), \tilde{R}_{a}\left(t, t^{\prime}\right)=\tilde{R}\left(t \neq t^{\prime}\right), \tilde{m}_{a}^{x}(t)=\tilde{m}^{x}
\end{aligned}
$$

Under the RS ansatz and the static approximation, $e^{G_{1}}$ is represented as

$$
e^{G 1} \equiv \exp \left\{\beta n N\left(s J_{0} m^{p}+\frac{\beta s^{2} J^{2}}{4}\left((n-1) q^{p}+R^{p}\right)+\mathcal{O}\left(\frac{1}{M}\right)\right)\right\} .
$$

We compute $e^{G 2}$ under the RS ansatz and the static approximation as follows:

$$
\begin{aligned}
e^{G_{2}} & =\sum_{\left\{\xi_{i}= \pm 1\right\}} \sum_{\left\{\tau_{i}= \pm \xi_{i}\right\}} P(\xi) P(\boldsymbol{\tau}) \operatorname{Tr} \exp \left\{\frac{\beta \tilde{m}}{M} \sum_{a, t, i} \sigma_{i a}^{z}(t)\right. \\
& +\frac{\beta(1-\lambda)(1-s)}{M} \sum_{a, t, i} \tau_{i} \xi_{i} \sigma_{i a}^{z}(t)+\frac{\beta^{2} \tilde{R}}{2 M^{2}} \sum_{a, t \neq t^{\prime}} \sum_{i=1}^{N} \sigma_{i a}^{z}(t) \sigma_{i a}^{z}\left(t^{\prime}\right) \\
& \left.+\frac{\beta^{2} \tilde{q}}{2 M^{2}} \sum_{a \neq b} \sum_{t, t^{\prime}} \sum_{i=1}^{N} \sigma_{i a}^{z}(t) \sigma_{i b}^{z}\left(t^{\prime}\right)+\frac{\beta \tilde{m}^{x}}{M} \sum_{a, t} \sum_{i=1}^{N} \sigma_{i a}^{x}(t)\right\} \\
& \times \prod_{a, t, i}\left\langle\sigma_{i a}^{z}(t) \mid \sigma_{i a}^{x}(t)\right\rangle\left\langle\sigma_{i a}^{x}(t) \mid \sigma_{i a}^{z}(t+1)\right\rangle \\
& =\prod_{i=1}^{N} \sum_{\xi_{i}= \pm 1} \sum_{\tau_{i}= \pm \xi_{i}} \frac{1}{2} P\left(\tau_{i}\right) \int D z \prod_{a=1}^{n} D y \prod_{t=1}^{M} \operatorname{Tr} \exp \left\{\frac{\beta}{M}(\tilde{m}\right. \\
& \left.+(1-s)(1-\lambda) \tau_{i} \xi_{i}+\tilde{q} z+\sqrt{\tilde{R}-\tilde{q} y)} \sigma_{i a}^{z}(t)+\frac{\beta \tilde{m}^{x}}{M} \sigma_{i a}^{x}(t)\right\} \\
& \times \prod_{a, t, i}\left\langle\sigma_{i a}^{z}(t) \mid \sigma_{i a}^{x}(t)\right\rangle\left\langle\sigma_{i a}^{x}(t) \mid \sigma_{i a}^{z}(t+1)\right\rangle \\
& =\prod_{i=1}^{N} \sum_{\xi_{i}= \pm 1} \sum_{\tau_{i}= \pm \xi_{i}} \frac{1}{2} P\left(\tau_{i}\right) \int D z\left(\int D y 2 \cosh \beta \sqrt{g^{2}\left(\tau_{i}, \xi_{i}\right)+\left(\tilde{m}^{x}\right)^{2}}\right)^{n}
\end{aligned}
$$




$$
\begin{aligned}
& \simeq \prod_{i=1}^{N} \sum_{\xi_{i}= \pm 1} \frac{1}{2} \exp \left\{n \int D z \sum_{\tau_{i}= \pm \xi_{i}} P\left(\tau_{i}\right) \ln \int D y 2 \cosh \beta \sqrt{g^{2}\left(\tau_{i}, \xi_{i}\right)+\left(\tilde{m}^{x}\right)^{2}}\right\} \\
& =\exp \left\{n N\left(\sum_{a= \pm 1} c_{a} \int D z \ln \int D y 2 \cosh \beta \sqrt{g_{a}^{2}+\left(\tilde{m}^{x}\right)^{2}}\right)\right\}
\end{aligned}
$$

where

$$
\begin{aligned}
g\left(\tau_{i}, \xi_{i}\right) & =\tilde{m}+(1-s)(1-\lambda) \tau_{i} \xi_{i}+\sqrt{\tilde{q}} z+\sqrt{\tilde{R}-\tilde{q}} y \\
g_{a} & =\tilde{m}+a(1-s)(1-\lambda)+\sqrt{\tilde{q}} z+\sqrt{\tilde{R}-\tilde{q}} y
\end{aligned}
$$

We apply the Hubbard-Stratonovich transformation,

$$
\exp \left(\frac{x^{2}}{2}\right)=\int D v_{1} \exp \left(x v_{1}\right)
$$

to the terms $\left(\beta \sqrt{\tilde{q}} / M \sum_{a, t} \sigma_{i a}^{z}(t)\right)^{2} / 2$ and $\sum_{a}\left(\beta \sqrt{\tilde{R}-\tilde{q}} / M \sum_{t} \sigma_{i a}^{z}(t)\right)^{2} / 2$. We now perform the inverse operation of the Suzuki-Trotter decomposition and take the trace.

Under the RS ansatz and the static approximation, $e^{G_{3}}$ is expressed as

$$
e^{G_{3}}=\exp \left\{\beta n N\left(-m \tilde{m}-m^{x} \tilde{m}^{x}-\frac{\beta}{2} R \tilde{R}-\frac{\beta(n-1)}{2} q \tilde{q}+(1-s) \lambda m^{x}\right)+\mathcal{O}\left(\frac{1}{M}\right)\right\} .
$$

In the thermodynamic limit $N \rightarrow \infty$, the saddle-point method can be used. The RS free energy density is then expressed as

$$
\begin{aligned}
-\beta f_{\mathrm{RS}}= & \lim _{n \rightarrow 0} \frac{\left[Z^{n}\right]-1}{n N} \\
= & \operatorname{extr}_{\substack{m, q, R \\
\tilde{m}, \tilde{q}, \tilde{R}}}\left[\beta s J_{0} m^{p}+\frac{\beta^{2} s^{2} J^{2}}{4}\left(R^{p}-q^{p}\right)+\beta(1-s) \lambda m^{x}-\beta m \tilde{m}-\beta m^{x} \tilde{m}^{x}\right. \\
- & \left.\frac{\beta^{2}}{2}(R \tilde{R}-q \tilde{q})+\sum_{a= \pm 1} c_{a} \int D z \ln \int D y 2 \cosh \beta \sqrt{g_{a}^{2}+\left(\tilde{m}^{x}\right)^{2}}\right] .
\end{aligned}
$$

The order parameters and their auxiliary parameters can be determined from the saddle-point conditions. 


\section{References}

1. J.R.L. de Almeida, D.J. Thouless, Stability of the Sherrington-Kirkpatrick solution of a spin glass model. J. Phys. A Math. Gen. 11(5), 983-990 (1978)

2. S. Arai, M. Ohzeki, K. Tanaka, Mean field analysis of reverse annealing for code-division multiple-access multiuser detection. Phys. Rev. Res. 3 (2021)

3. E. Farhi, J. Goldstone, S. Gutmann, J. Lapan, A. Lundgren, D. Preda, A quantum adiabatic evolution algorithm applied to random instances of an np-complete problem. Science 292(5516), $472-475$ (2001)

4. P. Gillin, H. Nishimori, D. Sherrington, Multispin Ising spin glasses with ferromagnetic interactions. J. Phys. A Math. Gen. 34(14), 2949-2964 (2001)

5. J.J. Hopfield, Neural networks and physical systems with emergent collective computational abilities. Proce. Nat. Acad. Sci. 79(8), 2554-2558 (1982)

6. J.I. Inoue, Application of the quantum spin glass theory to image restoration. Phys. Rev. E $\mathbf{6 3}$, $046114(2001)$

7. M.W. Johnson, P. Bunyk, F. Maibaum, E. Tolkacheva, A.J. Berkley, E.M. Chapple, R. Harris, J. Johansson, T. Lanting, I. Perminov, E. Ladizinsky, T. Oh, G. Rose, A scalable control system for a superconducting adiabatic quantum optimization processor. Supercond. Sci. Technol. 23(6) (2010)

8. Y. Kabashima, A CDMA multiuser detection algorithm on the basis of belief propagation. J. Phys. A: Math. Gen. 36(43), 11111-11121 (2003)

9. T. Kadowaki, H. Nishimori, Quantum annealing in the transverse ising model. Phys. Rev. E 58, 5355-5363 (1998)

10. S. Matsuura, H. Nishimori, T. Albash, D.A. Lidar, Mean field analysis of quantum annealing correction. Phys. Rev. Lett. 116 (2016)

11. F. Neukart, G. Compostella, C. Seidel, D. von Dollen, S. Yarkoni, B. Parney, Traffic flow optimization using a quantum annealer. Frontiers in ICT 4, 29 (2017)

12. H. Nishimori, Statistical Physics of Spin Glasses and Information Processing: An Introduction (Oxford University Press, Oxford, 2001)

13. M. Ohkuwa, H. Nishimori, D.A. Lidar, Reverse annealing for the fully connected $p$-spin model. Phys. Rev. A 98 (2018)

14. M. Ohzeki, A. Miki, M.J. Miyama, M. Terabe, Control of automated guided vehicles without collision by quantum annealer and digital devices. Front. Comput. Sci. 1, 9 (2019)

15. Y. Otsubo, J.I. Inoue, K. Nagata, K., Okada, M.: Effect of quantum fluctuation in errorcorrecting codes. Phys. Rev. E 86, 051138 (2012)

16. Y. Otsubo, J.I. Inoue, K. Nagata, M. Okada, Code-division multiple-access multiuser demodulator by using quantum fluctuations. Phys. Rev. E 90, 012126 (2014)

17. A. Perdomo-Ortiz, S.E. Venegas-Andraca, A. Aspuru-Guzik, A study of heuristic guesses for adiabatic quantum computation. Quant. Inf. Process. 10(1), 33-52 (2011)

18. Y. Seki, H. Nishimori, Quantum annealing with antiferromagnetic transverse interactions for the hopfield model. J. Phys. A: Math. Theor. 48(33) (2015)

19. B. Seoane, H. Nishimori, Many-body transverse interactions in the quantum annealing of thepspin ferromagnet. J. Phys. A Math. Theor. 45(43) (2012)

20. D. Sherrington, S. Kirkpatrick, Solvable model of a spin-glass. Phys. Rev. Lett. 35, 1792-1796 (1975)

21. N. Sourlas, Spin-glass models as error-correcting codes. Nature 339, 693 EP (1989)

22. M. Suzuki, Generalized trotter's formula and systematic approximants of exponential operators and inner derivations with applications to many-body problems. Commun. Math. Phys. 51(2), 183-190 (1976)

23. S. Suzuki, M. Okada, Residual energies after slow quantum annealing. J. Phys. Soc. Jpn 74(6), 1649-1652 (2005)

24. T. Tanaka, Statistical mechanics of CDMA multiuser demodulation. EPL (Europhysics Letters) 54(4), 540 (2001) 
25. D. Thirumalai, Q. Li, T.R. Kirkpatrick, Infinite-range ising spin glass in a transverse field. J. Phys. A Math. Gener. 22(16), 3339-3349 (1989)

26. Y. Yamashiro, M. Ohkuwa, H. Nishimori, D.A. Lidar, Dynamics of reverse annealing for the fully connected $p$-spin model. Phys. Rev. A 100, (2019)

Open Access This chapter is licensed under the terms of the Creative Commons Attribution 4.0 International License (http://creativecommons.org/licenses/by/4.0/), which permits use, sharing, adaptation, distribution and reproduction in any medium or format, as long as you give appropriate credit to the original author(s) and the source, provide a link to the Creative Commons license and indicate if changes were made.

The images or other third party material in this chapter are included in the chapter's Creative Commons license, unless indicated otherwise in a credit line to the material. If material is not included in the chapter's Creative Commons license and your intended use is not permitted by statutory regulation or exceeds the permitted use, you will need to obtain permission directly from the copyright holder.

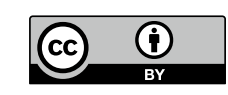

